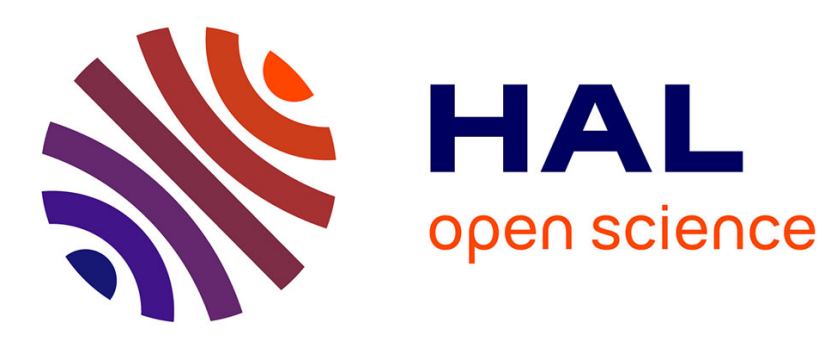

\title{
Dynamic Cell-Less Radio Access Network Meta-Scheduler for High System Capacity Increase
}

Christopher Merlhe, Cédric Gueguen

\section{To cite this version:}

Christopher Merlhe, Cédric Gueguen. Dynamic Cell-Less Radio Access Network Meta-Scheduler for High System Capacity Increase. 21st IEEE International Symposium on a World of Wireless, Mobile and Multimedia Networks, Aug 2020, Cork, Ireland. 10.1109/WoWMoM49955.2020.00031 . hal03052657

\section{HAL Id: hal-03052657 https://hal.science/hal-03052657}

Submitted on 19 Jan 2021

HAL is a multi-disciplinary open access archive for the deposit and dissemination of scientific research documents, whether they are published or not. The documents may come from teaching and research institutions in France or abroad, or from public or private research centers.
L'archive ouverte pluridisciplinaire HAL, est destinée au dépôt et à la diffusion de documents scientifiques de niveau recherche, publiés ou non, émanant des établissements d'enseignement et de recherche français ou étrangers, des laboratoires publics ou privés. 


\section{Dynamic Cell-Less Radio Access Network Meta-Scheduler for High System Capacity Increase}

\author{
Christopher Merlhe \\ University of Rennes 1, IRISA \\ Campus de Beaulieu \\ 35042 Rennes \\ christopher.merlhe@irisa.fr
}

\author{
Cedric Gueguen \\ University of Rennes 1, IRISA \\ Campus de Beaulieu \\ 35042 Rennes \\ cedric.gueguen@irisa.fr
}

\begin{abstract}
Radio resources are limited and subject to interferences, multi-path fading, shadowing and path-loss which highly impact the transmission efficiency. Optimizing the usage of these resources is the main issue in wireless networks. The resource management can be performed at different levels: in intracellular context, it is carried out by scheduling algorithms while in multi-cellular context, it is done by interference management strategies. This paper aims to merge these two steps. The proposed solution is compatible with the most acknowledged schedulers in order to optimize spectrum usage in intra-cell domain while efficiently decreasing the interferences in multicell domain. An innovative strategy is to consider the network as an "hyper-cell" rather than a sum of cells quite independent. This strategy often called "Cell-less" is especially effective in a Cloud Radio Access Network (C-RAN) architecture because it allows to centralize the decision making. This is more suitable for interference management as it provides a better flexibility to the system by retrieving and managing data from several cells that allows an overall performance increase. The proposed solution called Dynamic Cell-less Radio Access Network MetaScheduler (DC-RAN-MS) dynamically handles for each cell the management of radio resources depending on the interferences potentially experienced by users. Performance evaluation shows that the DC-RAN-MS offers an increased system capacity by optimizing the usage of bandwidth while reducing the magnitude of interferences received.
\end{abstract}

Index Terms-Wireless Network, Interference management, Cell-less, C-RAN, Opportunistic Scheduling.

\section{INTRODUCTION}

Guaranteeing a good Quality of Service (QoS) and Quality of Experience $(\mathrm{QoE})$ to the users is challenging in wireless networks. The continuously growing needs in term of throughput, system capacity and delay requirements require to optimize transmission efficiency particularly in ultra dense networks. In this way, scheduling algorithms are a crucial issue to achieve this goal.

Traditional solutions like Round Robin (RR) [1,2] and Random Access (RA) schedulers were inherited from the wired context. These solutions do not take into account the physical layer information. Thus, they neglect the channel state attenuations due to the path loss, the shadowing and the multipath fading. This last induces high variations of throughput over the time and on each sub-carrier. Consequently, RR and
RA solutions provide a poor throughput and system capacity, making them unsuitable for the wireless network specificities [1].

Consequently, many works have been done to design opportunistic scheduling algorithms (i.e. using the physical layer information). For instance, Maximum Signal to Noise Ratio (MaxSNR) takes benefits from the multi-user and frequency diversities by allocating the Resource Units (RUs) to mobiles with the best Signal-to-Noise Ratio (SNR) [3,4]. Its good throughput, high system capacity and its ease of implementation make it one of the most acknowledged scheduler.

However, its unfair behavior regarding the distance of mobiles from the access point (due to the path loss) leads to develop different resource allocation algorithms like the Proportional Fair (PF) and PF-based schedulers [1, 5-7]. These scheduling algorithms perform a fairer resource allocation which increases the global QoS of the system.

Nevertheless, these solutions have the similarity of being built for an intra-cellular context. It means that they does not manage efficiently Inter-Cell Interference (ICI). In this way, interference management solutions are another level of the performance control. Depending on the interference management algorithm chosen, the global QoS of a system can highly vary. Classical approaches like Reuse 1 and Sliced Bandwidth (for instance the Reuse 3) [8], make cells to squarely ignore interference management or to limit the usage of their bandwidth. Usually, with these classical solutions, cells are managed independently of each other. The Reuse 1 solution uses the completeness of the bandwidth without avoiding ICI. This scheme uses all the available resources but the interference impacts on the radio transmission quality is high, especially for mobiles in cell edges. Thus, the most distant mobiles from their access point will be highly penalized considering their poor SNR (due to the path loss) and the magnitude of interferences received (regarding their proximity to the neighboring cells).

The classical ICI avoidance solution is the Sliced Bandwidth. This scheme splits its frequency band according to the number of neighboring cells. In this way, there is no interference and the mobiles in cell edges are protected. However, there is a high unused bandwidth leading to a poor system capacity. 


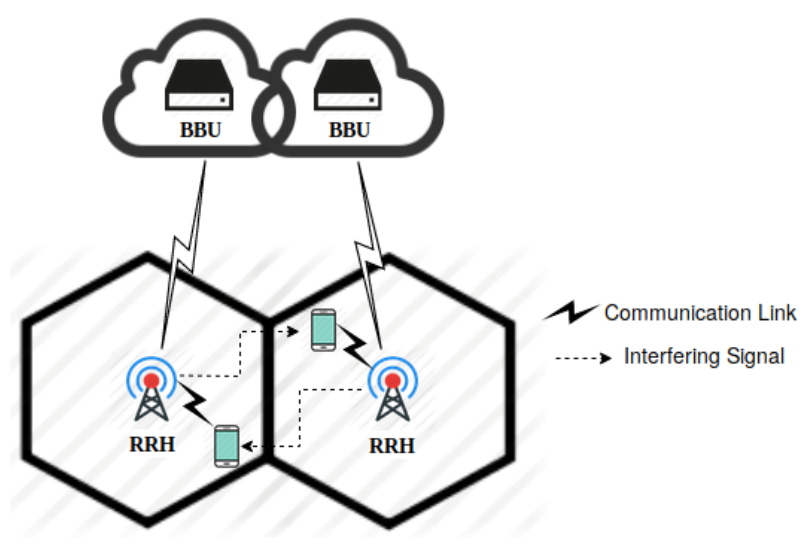

(a) One-to-one logical mapping.

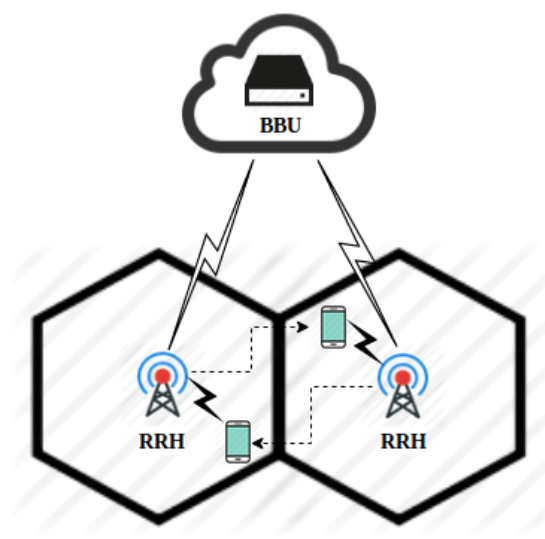

(b) One-to-many mapping.

Fig. 1. Example of C-RAN approaches

In $[9,10]$, the Fractional Frequency Reuse (FFR) and Soft Frequency Reuse (SFR) solutions are presented. These classical approaches perform a Sliced Bandwidth strategy in cell edges while in inner cell, a Reuse 1 strategy is used. In addition, SFR solution provides different power levels between the inner cell and edges, leading to a better usage of the bandwidth. Despite that the performances are increased compared to Reuse 1 and Sliced Bandwidth solutions, SFR and FFR schemes can not adapt their behavior to the mobile distribution as frequencies allocated to each cell are fixed beforehand.

All these classical solutions have a static behavior and perform their ICI only according to the mobile locations. Although the decision attributing resource blocks is taken in multi-cell domain, the allocation is performed in intracell domain which prevents the processing of special cases. For instance, they do not take into account the Signal-toInterference-plus-Noise Ratio (SINR) as well as the information that a mobile could be not interfered at a given time. This leads to inaccurate allocations and a poor flexibility regarding the channel state and the mobile distribution in the cell. Thus, designing an interference management approach in a C-RAN architecture seems to be more effective as it allows to centralize the decision making and to retrieve data from several access points.

Traditionally in C-RAN architecture [11,12], base stations are split into three parts : the Remote Radio Head (RRH), the Baseband Unit (BBU) and the optical link with highperformance, low delay and high bandwidth front-haul which connects the RRH to the cloud pool where BBUs are gathered. In C-RAN, most of the computational tasks can be considered as moved to BBU pool such as the channel state information estimation, the centralized signal processing management of the RAN etc [13]. On the other hand, the RRHs include radio antennas with their associated amplifier and are dispatched among several remote sites [14]. Thus, It is possible to distinguish different C-RAN oriented approaches.

The first is the one-to-one logical mapping (Fig.1(a)) where one $\mathrm{BBU}$ is assigned to one RRH. Although BBUs are gathered in the same BBU pool which can reduce the maintenance costs, this mapping only allows to manage one RRH at the same time. This makes ICI management solutions to have a static behavior. Consequently, it is not suitable to react to the channel state variations and to the user mobility.

Another approach is the one-to-many mapping (Fig.1(b)), where one BBU is assigned to several RRHs. It allows to open new prospects. For instance, an innovative approach is to consider the network as "Cell-less" $[15,16]$. The network is seen as an "hyper-cell" rather than a sum of independent cells. The cells become a set of access points and the decision making for this cluster is fully centralized. In this way, the "Cell-less" approach with the one-to-many mapping enables to manage several cells with only one entity. Thus, ICI can be fully controlled in a multi-cellular oriented approach which allows to share frames between RRHs providing a better usage of the bandwidth while efficiently reducing the magnitude of interferences received.

In [14], the Hybrid Static (HS) strategy is presented. It is based on the analyze that giving resources to mobiles from different cells, at the same time and on the same frequency is better than protected them when the magnitude of interferences received is not significant. Thus, the HS splits its bandwidth into two slices. In the first slice, a Reuse 1 strategy is used and it is allocated to mobiles in inner cell. On the other hand, the second slice is allocated to mobiles in cell edges where a Sliced Bandwidth strategy is performed. In this slice, the HS compares the channel state of mobiles chosen by the intracell scheduler and allocates the resource to the one with the best SNR. Thus, this slice is fully shared between the RRHs which allows to optimize the resource allocation regardless of the number of mobiles in the edges of cells considered. Consequently, the HS solution makes a better usage of the spectrum than SFR/FFR approaches. However, with a static boundary between the two slices, the HS solution relies on an almost evenly distribution of mobiles between the inner and the edges of the cell. This makes this solution to have a static behavior which can hardly adapt to the context. Moreover, depending on the mobile distribution, one slice reaches the congestion before the other. 
This paper aims to make a better usage of the one-to-many mapping by proposing the Dynamic Cell-less Radio Access Network Meta-Scheduler (DC-RAN-MS). The DC-RAN-MS relies on the following analysis:

- ICI management performed in intra-cell domain often induces a waste of bandwidth due to frequencies allocated beforehand. For instance, classical solution does not take into account that a mobile could not be interfered at a given time.

- Static solutions can not react to mobile distribution and to the channel state. In this way, they neglect that mobiles in cell edges could have an enough significant SINR to perform a Reuse 1 strategy (and conversely).

Consequently, the ICI management should coordinate with the schedulers in order to provide a more accurate resource allocation. Thus, the DC-RAN-MS aims to merge the intra-cell scheduling and the ICI management by controlling a cluster of RRHs. The DC-RAN-MS acts as a meta-scheduler by allowing or preventing schedulers to allocate resources according to: the channel state (i.e. the SINR/SNR), the magnitude of interferences experienced and the number of mobiles available to transmit in each RRH. Unlike the previously described solutions, the usage of a Reuse 1 or Sliced Bandwidth strategy does not rely on the position of mobiles in cells but on interferences themselves. The entire bandwidth is shared between the cluster of RRHs considered and resources unallocated by a RRH can be used by another. This provides a more accurate and dynamic ICI management which reduces the bandwidth waste and the magnitude of interferences experienced.

This paper is organized as follow: section II describes the proposed solution and performance evaluation is explained in section III. This paper ends with the conclusion in section IV.

\section{Dynamic Cell-Less Radio Access Network META-SCHEDULER (DC-RAN-MS)}

\section{A. Cell-Less model}

In this work, the global available bandwidth is assumed divided in sub-frequency bands called sub-carriers. The radio resources are distributed in the time domain in frames where each frame is divided in Time Slot (TS) of constant duration. A Resource Unit is defined as a pair (sub-carrier, time slot). The channel gain between the RRH $i$ and the user $k$ on the sub-carrier $n$ is given by :

$$
G_{k, n}^{i}=h \times 10^{\frac{X \sigma}{10}} \times\left(\frac{1}{d_{k, i}}\right)^{\alpha}
$$

where $h$ represents the Rayleigh multi-path fading, which is modeled by and exponential distribution, $\mathrm{X}$ is a standard Gaussian random variable, $\sigma$ is the standard derivation of shadowing in $\mathrm{dB}, \mathrm{d}_{k, i}$ is the distance between the mobile $k$ and the RRH i and $\alpha$ is the path loss exponent. We denote $C$, the set of $L$ neighboring RRHs of the RRH $i$ considered mapped to the BBU of the DC-RAN. Thus, the Signal-to-Interference-
plus-Noise Ratio of user $k$ on sub-carrier $n$ associated to RRH $i$ is given by :

$$
\gamma_{k, n}^{i}=\frac{P_{n}^{i} G_{k, n}^{i}}{B_{s u b} N_{0}+\sum_{(j=1, j \in C)}^{L} P_{n}^{j} G_{k, n}^{j}}
$$

where $\mathrm{P}_{n}^{i}$ and $\mathrm{P}_{n}^{j}$ are respectively the transmitted power on sub-carrier $n$ of RRH $i$ and the transmitted power on subcarrier $n$ of the interfering RRH $j$ that belongs to the set $C$. $\mathrm{G}_{k, n}^{j}$ is the channel gain between the mobile $k$ and RRH $j$, the parameter $\mathrm{N}_{0}$ is the thermal noise power density, and $\mathrm{B}_{\text {sub }}$ is the sub-carrier spacing [17].

To compute the spectral efficiency $\eta_{k, n}$ of mobile $k$ on subcarrier $n$ associated to RRH $i$, the Shannon's formula is used such as:

$$
\eta_{k, n}^{i}=\log _{2}\left(1+\frac{\gamma_{k, n}^{i}}{\Gamma}\right)
$$

with $\Gamma$, a SNR correction factor that takes into account the difference between the information-theoretic performances and the practical implementation of the MCS [18] defined as follows:

$$
\Gamma=-\frac{\ln (5 . E)}{1.5}
$$

where $\mathrm{E}$ is a BER Target.

\section{B. The proposed solution}

The DC-RAN-MS relies on a one-to-many mapping within the scope of a "Cell-less" approach. In this way, it manages several RRHs at the same time and aims to coordinate with the intra-cell schedulers. The proposed solution is compatible with the most acknowledged schedulers while emphasizing their specific features (throughput and system capacity for the MaxSNR, system capacity and fairness for the PF etc.). The mobile $m$ is defined as the mobile chosen by the scheduler on the sub-carrier $n$ in the RRH $i$. For instance, with a MaxSNR allocation, $m$ is computed such as:

$$
m=\underset{k}{\operatorname{argmax}}\left(\eta_{k, n}^{i}\right)
$$

The DC-RAN-MS avoids ICI when their magnitude is high by restricting the usage of a sub-carrier $n$ for a given TS. Otherwise, the proposed solution uses the entirety of the spectrum. This decision is taken according to the channel state of the mobile $m$ in each RRH. The $\eta_{m, n_{\text {interf }}}^{i}$ is defined as the $\eta_{m, n}^{i}$ while the mobile $m$ of the RRH $i$ is interfered on the sub-carrier $n$. In this way, the DC-RAN-MS performs its resource allocation following this inequality:

$$
\sum_{(i=1, i \in C)}^{L} \eta_{m, n_{\text {interf }}}^{i} \geq \max \left\{\eta_{m, n}^{i}, \forall i \in C\right\}
$$

Thanks to this inequality, the DC-RAN-MS can deduce the number of mobiles available to transmit (i.e. if they are interfered). Depending on their channel states, the proposed solution performs different frequency reuses. When there is no interference or their magnitudes are too high the DCRAN-MS uses a Sliced Bandwidth strategy. Otherwise, if the 


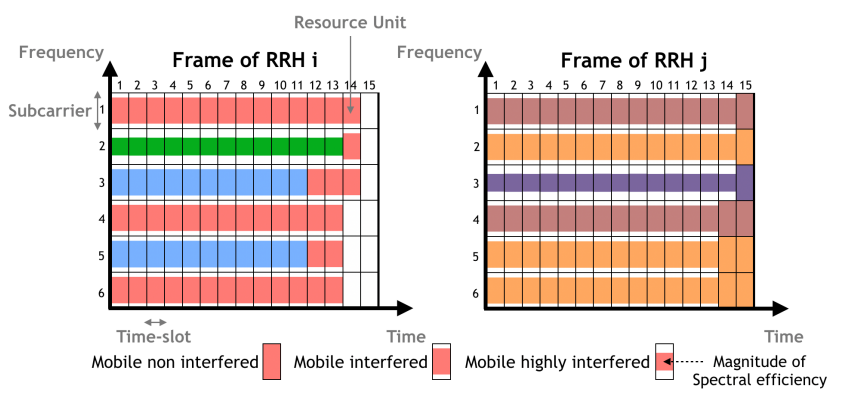

(a) Reuse 1 strategy.

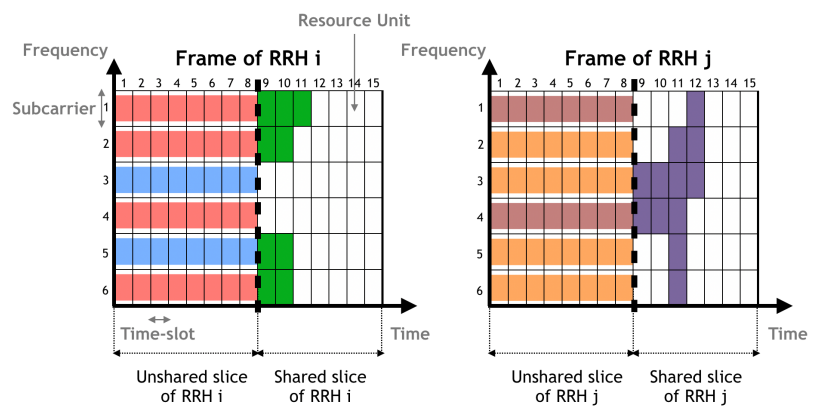

(c) Hybrid Static strategy.

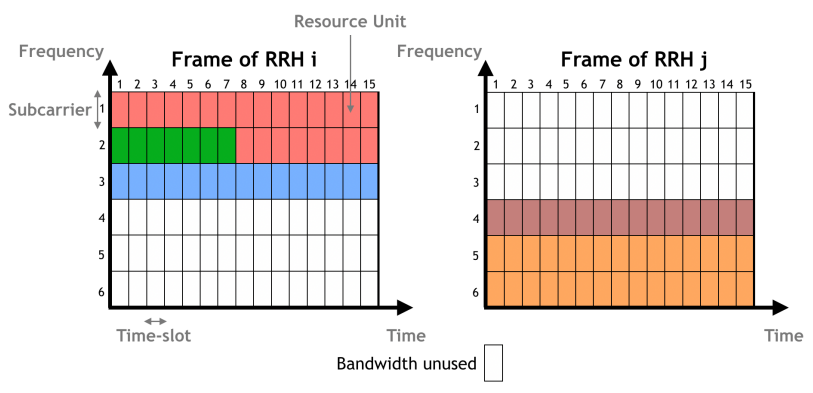

(b) Sliced Bandwidth strategy.

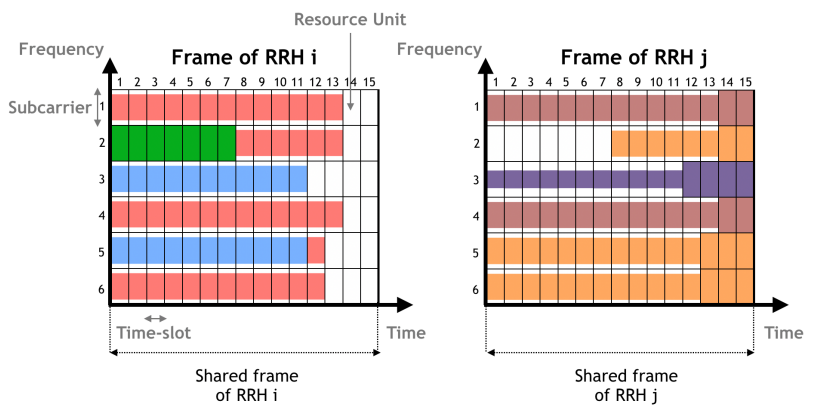

(d) DC-RAN-MS strategy.

Fig. 2. Interference management solutions with a MaxSNR allocation.

interferences are not significant, it performs a Reuse 1 scheme. Consequently, the DC-RAN-MS does not rely on a criteria in correlation with interferences (i.e the distance like state of the art solutions) but directly on the interferences themselves.

\section{Operation of the DC-RAN-MS}

The figure 2 illustrates some solutions previously described. For each solution, we consider two frames belonging respectively to the RRH $i$ and the RRH $j$. Within the RRHs, the scheduling algorithm performed is a MaxSNR. The RUs are allocated TS per TS and denoted by (sub-carrier, Time Slot). A mobile is embodied by a specified color. The rate of color filling illustrates the spectral efficiency variations regarding the interferences received. To provide a realistic scenario, the mobiles have different needs in term of application requirements which leads to different RU allocations between the mobiles. The green and purple mobiles are considered in cell edges while the others are in the inner. In addition, the red mobile has the most packets to transmit.

The Reuse 1 strategy (Fig.2(a)) uses the entirety of the bandwidth without attempting to avoid ICI. Consequently, the green and purple mobiles are highly interfered and their spectral efficiency is significantly downgraded. This makes them to need much more RUs than usual to end their transmission. As the resource allocation is performed TS per TS in this example, the red mobile takes advantage that others mobiles have ended their transmission to transmit on the sub-carriers they previously used (RUs : $(3,12),(5,12),(3,13),(5,13)$, $(2,14)$ and ends on the RU $(3,14))$. However, considering that the red mobile is likely to do not have a good SNR on these sub-carriers and interferes mobiles of the RRH $j$, it is not always efficient that the red uses these RUs. Depending on the channel state of this mobile and the number of RUs available, it could be more profitable for the system that the red waits for transmitting on sub-carriers 1 and 4 . Notice that users from the RRH $j$ are not interfered on the RUs $(4,14),(5,14)$ and $(6,14)$ as well as on the TS 15 since users from the RRH $i$ have ended their transmission.

The classical ICI avoidance Sliced Bandwidth strategy (Fig.2(b)), splits its bandwidth into two parts. The Sliced Bandwidth strategy protects the green mobile which is highly interfered. However, due to the MaxSNR allocation, the purple mobile does not have any resource as the channel state of brown and orange mobiles is better. In addition, the half of the bandwidth is unused on each RRH which leads to a poor system capacity.

The HS solution (Fig.2(c)) divides the bandwidth into two slices. The left slice is allocated to mobiles in inner cell where a Reuse 1 strategy is performed. In the right slice, a Sliced Bandwidth scheme is used to protect cell edge mobiles. On this side, the HS solution compares the $\eta_{\text {green,n }}^{i}$ with the $\eta_{\text {purple, } n}^{j}$ and allocates the resource on the sub-carrier $n$ to the mobile with the best value. Although this solution attempts to provide a better fairness among mobiles by protecting the most affected from interferences, its static behavior is not well suitable for wireless networks specificities to be efficient. In this example, the Reuse 1 slice is overloaded and the RUs unused in the Sliced bandwidth part can not be allocated to the Reuse 1 one. Indeed, this solution relies on an ideal distribution between mobiles in inner cell and cell edge ones in order to avoid a load asymmetry between the two slices. In addition, even with 


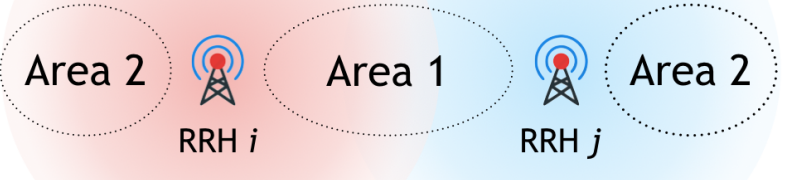

Fig. 3. Conceptual representation of the simulation context.

a moving boundary, as the radio conditions highly vary, it is not enough flexible to react to the channel state of mobiles and to their mobility.

In order to dynamically react to the context, the DC-RANMS performs an ICI management that does not rely on the locations of mobiles. The figure 2(d) illustrates the resource management of the proposed solution. For instance, in accordance with the inequality (6), the result of the ( $\eta_{\text {green, }}^{i} 2_{\text {interf }}$, $\eta_{\left.\text {orange }, 2_{\text {interf }}^{j}\right)}^{j}$ is not enough significant to perform a double allocation. In this way, as the green mobile has a better channel state than the orange, it starts to transmit on the RU $(2,1)$ and ends on the RU (2,7). On the other hand, the purple mobile also highly interfered, can transmit on the same TS and subcarrier than the blue as $\left(\eta_{\text {blue }, 3_{\text {interf }}}^{i}, \eta_{\text {purple }, 3_{\text {interf }}}^{j}\right)$ is greater than $\max \left\{\eta_{\text {blue }, 3}^{i}, \eta_{\text {purple }, 3}^{j}\right\}$. This allocation is not possible with state of the art solutions as they does not take into account the channel state but only the locations of mobiles. In addition, the red mobile takes advantage that blue and green mobiles have ended their transmission to use the following RUs: $(2,8)$ to $(2,13)$ and $(5,12)$. As the sum of $\eta_{\text {red, } n_{\text {interf }}}^{i}$ and $\eta_{\text {orange, } n_{\text {interf }}}^{j}$ on sub-carriers 2,5 and 6 is greater than their respective $\eta_{m, n}$, red and orange mobiles are allowed to transmit at the same time. However, red mobile can not transmit on the same TS than the purple (i.e. RU $(3,13))$ due to the high interferences experienced by this last and to the poor channel state.

To conclude, The DC-RAN-MS performs a wise resource allocation by providing a more accurate interference management according to: the possibility that a mobile could not be interfered, the magnitude of interferences experienced and their $\eta_{m, n}, \eta_{m, n_{\text {interf }}}$. This leads to an optimized decision making: either allocating the resources to all mobiles when the interferences are not significant or only allowing to one mobile to transmit when the channel state is poor. Thereby, the DC-RAN-MS optimizes the usage of the bandwidth, reduces the interferences experienced and protects the most affected users. This leads to increase the system capacity and the QoS.

\section{PERFORMANCES EVALUATION}

In this section, the DC-RAN-MS is compared to the classical C-RAN strategy, the HS solution, as well as to the classical
ICI management strategies: the Reuse 1 and Sliced Bandwidth schemes.

In the simulations, a small network of 2 adjacent cells is considered. The area 1 is a zone where the mobiles are likely to be highly interfered as they are closer to the neighboring cell than mobiles in area 2 (where the interferences are less significant). The figure 3 illustrates an example of the position of these areas. The scheduling algorithms of each cell are the same which allows to only study the influence of the interference management solutions. The scheduler chosen is the MaxSNR for its ability to increase the system capacity and as it is one of the most acknowledged scheduler. In addition, the mobiles are at the same distance from their respective RRH to neglect the unfair behavior of this scheduler. The traffic generated by sources is considered realistic and variable which produces high volume of data with important sporadic and tight delay requirements [19-21]. This significantly complicates the task of schedulers. Simulations parameters are described in the table I.

The performances evaluation is composed of 2 scenarios. For each scenario, only one parameter varies. The first examines when users are equally distributes between area 1 and 2 . Thus, the traffic load increases until the congestion with this particular distribution. On the other hand, the second scenario analyzes the performances of the different strategies when the repartition of mobile between the area 1 and 2 varies.

\begin{tabular}{|c|c|}
\hline Parameters & Value \\
\hline Cell Radius & $500 \mathrm{~m}$ \\
\hline Number of sub-carriers & 32 \\
\hline Number of Time Slots & 10 \\
\hline RRH transmit power & $20 \mathrm{~W}(43 \mathrm{dBm})$ \\
\hline Standard deviation of shadowing & $\sigma=8 \mathrm{~dB}$ \\
\hline Path-loss exponent & $3.5($ urban context) \\
\hline Target BER & $5 \times 10^{-5}$ \\
\hline Sub-carrier spacing & $15 \mathrm{kHz}$ \\
\hline Thermal noise power density $\left(\mathrm{N}_{0}\right)$ & $-174 \mathrm{dBm} / \mathrm{Hz}$ \\
\hline
\end{tabular}

TABLE I

SIMULATIONS PARAMETERS.

\section{A. Scenario 1 : Influence of the traffic load increase}

In this scenario, considering the two cells, the mobiles are equally distributed between the area 1 and 2 such as: $25 \%$ are located in area 2 of the RRH i, 50\% in the area 1 and $25 \%$ in area 2 of the RRH $j$. In this way, mobiles are added two per two in each cell (one per area). 


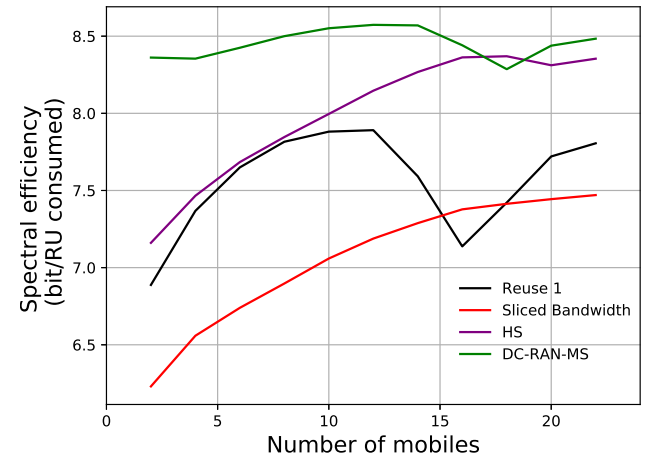

(a) Spectral efficiency.

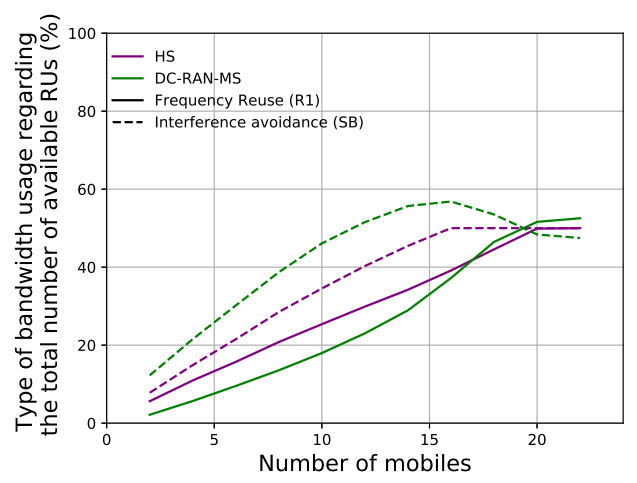

(c) DC-RAN-MS and HS behavior regarding the traffic load.

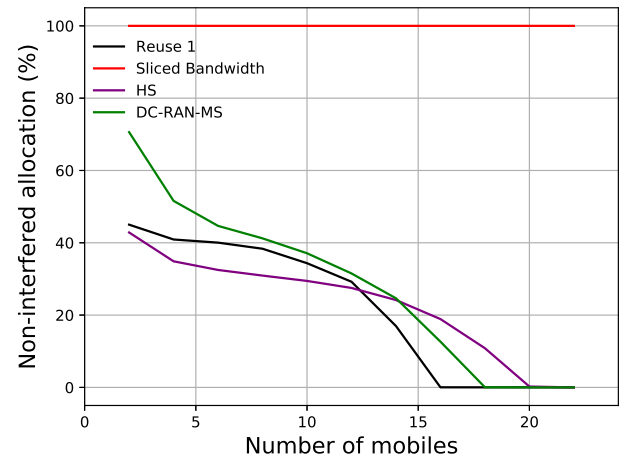

(b) Non-interfered allocation.

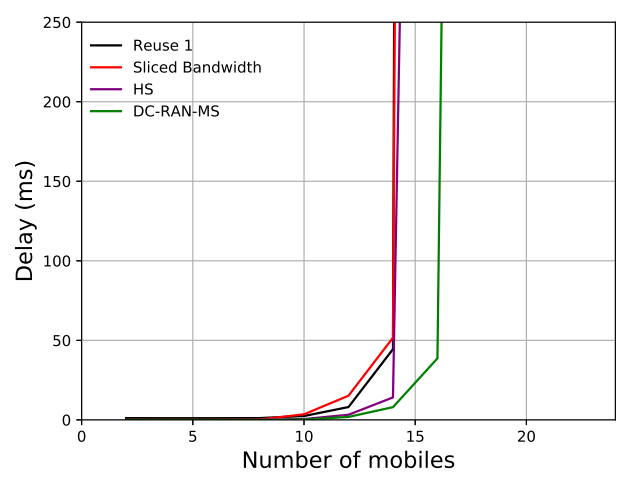

(d) Delay.

Fig. 4. Scenario 1.

1) Spectral efficiency: Figure 4(a) shows the spectral efficiency obtained with each solution for different traffic load in the system. The spectral efficiency presented takes into account the two cells considered. It means that it is the average of bit per RU consumed. A RU is considered as consumed either when it is used by the both RRHs or when an ICI management prevents a RRH from allocating the RU in order to protect a mobile in the other RRH. Computing the spectral efficiency only on RUs allocated is misleading because it does not take into account RUs unused to avoid interferences. For instance, given a RRH $i$ and a RRH $j$ which have only one mobile, respectively the mobile $m_{i}$ and the mobile $m_{j}$. In this example it is considered that they both get the same $\eta_{m, n}$ and $\eta_{m, n_{\text {interf }}}$ equal to 14 and 6.5. With a Reuse 1 solution their classical spectral efficiency (bits/RU) is equal to 6.5 while it is equal to 14 with a Sliced Bandwidth scheme as only the mobile $m_{i}$ is allowed to transmit (according to the frequency reuse). However, this last value does not take into account the RU of the RHH $j$ unused. To provide a more accurate indicator of the bandwidth waste, the average of bits per RU consumed seems to be more appropriate to compute the spectral efficiency. Thus, with the previous example, the spectral efficiency (bits/RU consumed) of $m_{i}$ and $m_{j}$ is equal to 6.5 with a Reuse 1 solution while for $m_{i}$ it is equal to 7 with a Sliced Bandwidth strategy $(14 \div 2$, where 2 is the number of RUs consumed).

The figure 4(b) shows the percentage of non-interfered allocation for different traffic load. An interference appears when a mobile of the RRH $i$ and a mobile of the RRH $j$ are chosen to transmit on the same frequency and time. In underloaded context, non-interfered allocations occur more frequently compared to a loaded system. Thus, this figure shows that the mobiles become more and more interfered as the traffic load increases. Indeed, it is rare that only one mobile is chosen to transmit on a given RU among the RRHs since their bandwidth become more and more filled. Notice that when the percentage of non-interfered allocation reaches zero, it means that there is no more RU available without being interfered.

The figure 4(c) shows the type of bandwidth usage regarding the total number of available RUs for different traffic load. It illustrates the usage ratio of the different ICI management (either Reuse 1 or Sliced Bandwidth) performed by the HS and DC-RAN-MS solutions.

On the figure 4(a), the solutions adopt at low traffic load, a typical behavior of a MaxSNR allocation which benefits from the multi-user and frequency diversities. In this way, their spectral efficiency increases with the traffic load. However, depending on the ICI management, interference effects on this metric highly vary. 
The Sliced Bandwidth avoids interferences and keeps the opportunistic MaxSNR behavior. Nevertheless, as the part of unused bandwidth is high, it provides a poor spectral efficiency. Notice that with a classical spectral efficiency computation (Bits/RU used) the result is significantly different.

From 12 users to 16 , the Reuse 1 solution experiences a high degradation of its spectral efficiency due to the decrease of the non-interfered allocation number (Fig.4(b)). At 16 users, the number of possible interferences has reached its peak: all the RUs are interfered and the system is overloaded. Since there are two phenomenons that face each other (the MaxSNR opportunistic behavior and the magnitude of interferences), from 16 users the MaxSNR allocation can counterbalance interference effects as it favors the less interfered users. This leads to increase the spectral efficiency of the Reuse 1 solution.

On the other hand, HS and DC-RAN-MS solutions are less affected by interferences as they provide a better ICI management.

The HS solution puts the cell edges mobiles in its right part (Fig.2(c)) where a Sliced Bandwidth is performed. Consequently, only users in inner cells are affected by interferences. As they are less interfered, the magnitude of ICI experienced is not significant making the spectral efficiency less affected by interferences (Fig.4(a)).

The DC-RAN-MS dynamically performs its ICI management thanks to appropriate allocations according to the channel state (Inequality (6)). In addition, unlike the HS solution, the DC-RAN-MS does not restrict the usage of the ICI management schemes (Reuse 1 or Sliced Bandwidth) to $50 \%$ of its bandwidth (Fig.4(c)). This provides a better usage of the bandwidth which leads to a high spectral efficiency (Fig 4(a)).

2) HS and DC-RAN-MS behaviors and system capacities: The system capacity provided by a solution does not only rely on its spectral efficiency. For instance, the HS solution has a better spectral efficiency at a traffic load of 18 mobiles (Fig 4(a)). As only mobiles in inner cell are affected by interferences, the percentage of mono allocation reaches $0 \%$ at a traffic load of 20 mobiles (Fig.4(b)). However, this is not relevant from the system capacity provided by the HS solution. The figure 4(c) shows that the interference avoidance (i.e. Sliced Bandwidth) part of the HS solution is overloaded since a while (it reaches $50 \%$ of the available bandwidth at a traffic load of 16 mobiles). Thus, as the boundary of the HS solution is static, it can not take advantage of the unused RUs in the Reuse 1 slice to allocate them to the Sliced Bandwidth part. This leads to an asymmetry load between mobiles in inner and edges of the cell.

Unlike the HS solution, the DC-RAN-MS can adapt its behavior to the context. When the system is underloaded, the DC-RAN-MS performs more Sliced Bandwidth allocation than Reuse 1 ones as only one mobile is allowed to transmit with this kind of frequency reuse (Fig.4(c)). As the DC-RANMS does not rely on a boundary, it can allocate the RUs unused by the Reuse 1 strategy to perform its Sliced Bandwidth allocations. This provides a better system capacity than the
HS solution for mobiles in inner cell and edges without an asymmetry.

3) Mean packet delay: A crucial indicator of the QoS experienced by users is the latency. Figure 4(d) represents the mean packet delay in the system in milliseconds.

The Sliced Bandwidth strategy provides a poor system capacity as a half of the bandwidth is unused. Thus, even if the mobiles are not interfered, they will quickly experience an increase of their mean packet delay due to the poor available RUs number as the traffic load rises.

The Reuse 1 solution uses the total available bandwidth. However, as there is no interference management, the most interfered mobiles are highly penalized which leads to provide a poor global QoS.

The HS strategy attempts to protect the most interfered mobiles by dividing the bandwidth. Nevertheless, as only one mobile is allowed to transmit on the Sliced Bandwidth part, this side is quickly overloaded (Fig.4(c)). As the boundary is static, the Reuse 1 part can not be used to provide a better system capacity and the RUs unused stays wasted until this part is overloaded too. Consequently it can better handle the QoS of mobiles than the two previously described solutions, but overall, the system collapses at the same traffic load than others solutions (14 mobiles).

The DC-RAN-MS optimizes the spectrum usage (Fig.4(a)) and allows to reduces the magnitude of interferences. The proposed solution performs a Sliced Bandwidth strategy only when its necessary (Fig.4(c)). This reduces the waste of bandwidth and provides a more accurate and fairer resource allocation. Consequently a better QoS is experienced and the system can handle the traffic load longer (16 users) than others solutions (Fig.4(d)).

\section{B. Scenario 2 : influence of users distribution}

This scenario analyzes the behavior of the solutions when users distribution varies between the areas 1 and 2 for a traffic load of 14 mobiles. First, mobiles are located in the area 1. Then they move one by one to the area 2 .

1) Spectral efficiency and system capacity: The Sliced Bandwidth strategy has a constant spectral efficiency (Fig.5(a)) as this solution manages the interferences by dividing its bandwidth. In this way, its spectral efficiency depends only on the traffic load and not on users distribution (i.e magnitude of interferences experienced)

The Reuse 1 solution provides a poor spectral efficiency (Fig.5(a)) when mobiles are highly interfered (i.e located in area 1). Thus, mobiles need much more RUs than usual to end their transmission which leads to consume all the RUs available and an overloaded system as the ratio of non-interfered allocation reaches $0 \%$ (Fig.5(b)). Like a virtuous circle, the rise of the spectral efficiency increases the percentage of non-interfered allocation. As the number of RUs interfered decreases, it provides a better spectral efficiency and so on.

The HS and DC-RAN-MS schemes provides a better ICI management leading to a better spectral efficiency. 


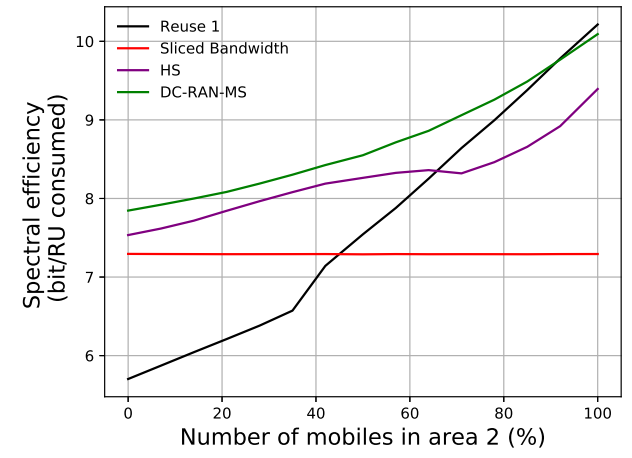

(a) Spectral efficiency.

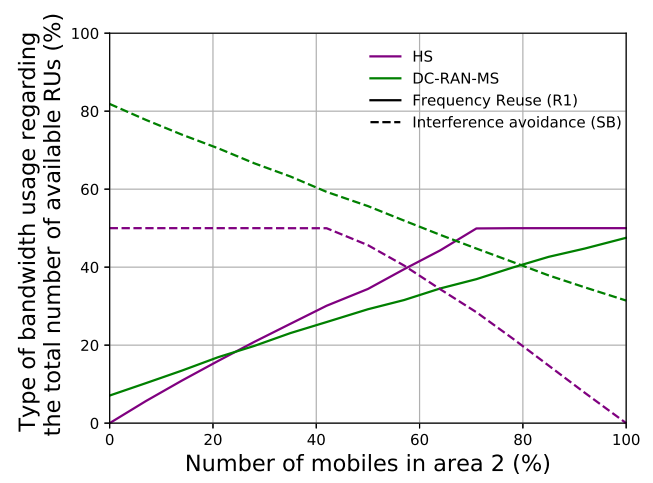

(c) DC-RAN-MS and HS behavior regarding the mobile distribution.

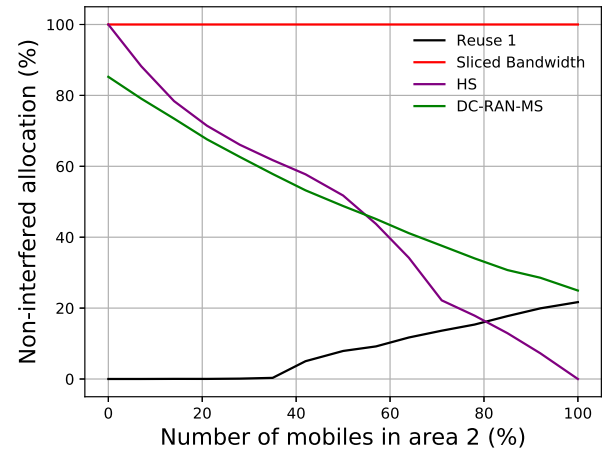

(b) Non-interfered allocation.

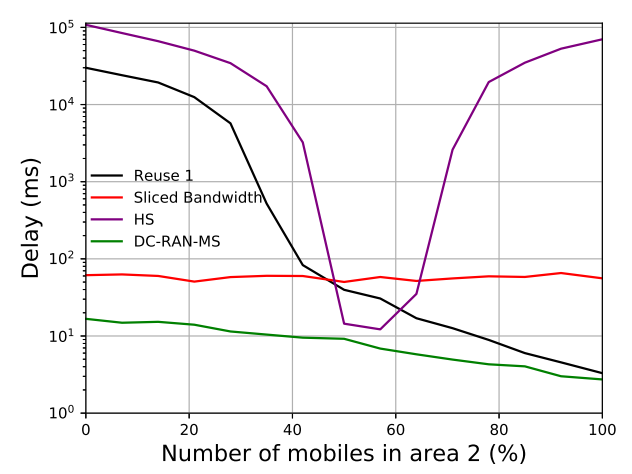

(d) Delay.

Fig. 5. Scenario 2

However, as the HS solution is static, there is an asymmetry between the Sliced Bandwidth part and the Reuse 1 one (Fig.5(c)). Until $42 \%$ of mobiles in the area 2, the Sliced Bandwidth part is overloaded and the spectral efficiency increases thanks to the opportunistic MaxSNR behavior. Then, the number of users interfered rises which decreases the percentage of non interfered allocation. At $71 \%$ of mobiles in area 2, the Reuse 1 slice is overloaded (Fig.5(c)) which means that the influence of interferences has reached its peak. Thus, the MaxSNR prioritizes less interfered mobiles and counterbalances the ICI effects. In this way, the spectral efficiency increases from $71 \%$ until $100 \%$ of mobiles in area 2. Notice that the percentage of non-interfered allocations reaches $0 \%$ only when mobiles are all located in area 2 as before, RUs are still allocated in the Sliced Bandwidth part.

Unlike the HS solution, the DC-RAN-MS has been designed to dynamically react to the context. The DC-RAN-MS does not take into account the location of mobiles but only their channel state. This allows to be even more accurate than a solution with a dynamic boundary. When the mobiles are mostly in the area 1 (i.e highly interfered) it uses mainly the Sliced Bandwidth strategy (Fig.4(c)). Nevertheless, it can performs a Reuse 1 strategy provided the radio conditions of mobiles are enough significant. The same analyze can be apply when mobiles are mostly located in area 2 (i.e. less interfered) where the percentage of Sliced bandwidth strategy used is still important. Consequently, the DC-RAN-MS provide a better spectral efficiency than other solutions (Fig.5(a)) and a better system capacity.

2) Mean packet delay: The Sliced bandwidth provides a constant delay (Fig.5(d)) as its spectral efficiency is the same whatever the distribution of mobiles.

The mean packet delay provided by the Reuse 1 solution highly depends on the mobile locations (i.e. magnitude of interference received). In this way, the mobiles have a better QoS when they are located in the area 2 than in the area 1.

The HS solution is designed with a static boundary. This leads to a poor QoS experienced by mobiles except when they have an ideal distribution (between $42 \%$ and $61 \%$ ).

The DC-RAN-MS dynamically adapt its behavior to the channel state of mobiles regardless of their location. Thanks to a better spectral efficiency and system capacity provided by the inequality (6), the proposed solution outperforms state of the art schemes on this metric. 


\section{CONCLUSION}

The continuously growing needs in term of throughput, system capacity and delay requirements lead to search new manner to optimize transmission efficiency particularly in ultra dense networks. In this way, the contribution of this paper named DC-RAN-MS, aims to merge the ICI management and the scheduling processes to optimize the resource allocation. Thanks to a "Cell-less" approach based on the C-RAN architecture, the proposed solution allocates resources for a cluster of RRHs. The proposed solution does not rely on a criteria in correlation with ICI (like the distance) but directly on the interferences themselves. According to the channel state of mobiles, the proposed solution performs dynamically its ICI management either by: allowing the usage of the entire bandwidth or by preventing schedulers from allocating resources when the magnitude of interferences experienced are too high. This leads to optimize the usage of the spectrum which decreases the global ratio of unused bandwidth while efficiently reducing the magnitude of interferences. This results in higher spectral efficiency, higher system capacity and a QoS increased.

\section{REFERENCES}

[1] M. Minelli, M. Ma, M. Coupechoux, and P. Godlewski, "Scheduling Impact on the Performance of Relay-Enhanced LTE-A Networks," IEEE Transactions on Vehicular Technology, vol. 65, no. 4, pp. 2496-2508, April 2016.

[2] A. Kuurne and A. Miettinen, "Weighted Round Robin scheduling strategies in (E)GPRS radio interface," in Proc. IEEE Int. Vehicular Technology Conference (VTC), vol. 5, Sept. 2004, pp. 3155 - 3159.

[3] C. Y. Wong and R. S. Cheng, "Multiuser OFDM with adaptive subcarrier, bit, and power allocation," IEEE J. Sel. Areas Commun., 1999.

[4] M. Bechir Dadi and R. Belgacem Chibani, "Scheduling performance's study for LTE downlink system," in 2017 IEEE International Conference on Green Energy Conversion Systems (GECS), March 2017, pp. 1-4.

[5] P. Viswanath, D. N. C. Tse, and R. Laroia, "Opportunistic beamforming using dumb antennas," IEEE Transactions on Information Theory, vol. 48, pp. 1277 - 1294, June 2002.

[6] H. Hamouda, M. O. Kabaou, and M. S. Bouhlel, "A Cross-Layer Downlink Scheduling Scheme for Balancing QoS in IEEE 802.16 Broadband Wireless Access Systems," in 2017 IEEE 86th Vehicular Technology Conference (VTC-Fall), Sep. 2017, pp. 1-5.

[7] X. Ge, H. Jin, and V. C. M. Leung, "Cdf-based scheduling algorithm for proportional throughput fairness," IEEE Communications Letters, vol. 20, no. 5, pp. 1034-1037, May 2016.

[8] S. Elayoubi, O. Ben Haddada, and B. Fourestie, "Performance evaluation of frequency planning schemes in OFDMA-based networks," IEEE Transactions on Wireless Communications, vol. 7, no. 5, pp. 1623-1633, May 2008.

[9] M. Qian, W. Hardjawana, Y. Li, B. Vucetic, X. Yang, and J. Shi, "Adaptive Soft Frequency Reuse Scheme for Wireless Cellular Networks," IEEE Transactions on Vehicular Technology, vol. 64, no. 1, pp. 118131, Jan 2015.

[10] S. Kumar, S. Kalyani, and K. Giridhar, "Impact of Sub-Band Correlation on SFR and Comparison of FFR and SFR," IEEE Transactions on Wireless Communications, vol. 15, no. 8, pp. 5156-5166, Aug 2016.

[11] A. Checko, "Cloud Radio Access Network architecture. Towards 5G mobile networks," Ph.D. dissertation, 2016.

[12] S. Park, O. Simeone, O. Sahin, and S. Shamai, "Robust and Efficient Distributed Compression for Cloud Radio Access Networks," IEEE Transactions on Vehicular Technology, vol. 62, no. 2, pp. 692-703, Feb 2013.

[13] K. Wang, K. Yang, and C. S. Magurawalage, "Joint Energy Minimization and Resource Allocation in C-RAN with Mobile Cloud," IEEE Transactions on Cloud Computing, vol. 6, no. 3, pp. 760-770, July 2018.
[14] M. Ezzaouia, C. Gueguen, M. E. Helou, M. Ammar, X. Lagrange, and A. Bouallegue, "A dynamic transmission strategy based on network slicing for Cloud Radio Access Networks," in 2018 Wireless Days (WD), April 2018, pp. 40-45.

[15] Q. Li, H. Niu, A. Papathanassiou, and G. Wu, "Edge Cloud and Underlay Networks: Empowering 5G Cell-Less Wireless Architecture," in European Wireless 2014; 20th European Wireless Conference, May 2014, pp. 1-6.

[16] T. Han, X. Ge, L. Wang, K. S. Kwak, Y. Han, and X. Liu, "5G Converged Cell-Less Communications in Smart Cities," IEEE Communications Magazine, vol. 55, no. 3, pp. 44-50, March 2017.

[17] M. Ezzaouia, C. Gueguen, M. YASSIN, M. Ammar, X. Lagrange, and A. Bouallegue, "Autonomous and Dynamic Inter-Cell Interference Coordination Techniques for Future Wireless Networks," in WiMob 2017 - 13th IEEE International Conference on Wireless and Mobile Computing, Networking and Communications, Rome, Italy, Oct. 2017.

[18] H. Seo and Byeong Gi Lee, "A proportional-fair power allocation scheme for fair and efficient multiuser OFDM systems," in IEEE Global Telecommunications Conference, 2004, vol. 6, Nov 2004, pp. 37373741.

[19] S. Tanwir and H. Perros, "A survey of VBR video traffic models," IEEE Communications Surveys Tutorials, vol. 15, no. 4, pp. 1778-1802, 2013.

[20] A. K. A. Tamimi, R. Jain, and C. So-In, "Modeling and prediction of high defninition video traffic: A real-world case study," in 2010 Second International Conferences on Advances in Multimedia, June 2010, pp. $168-173$.

[21] D. P. Heyman, "The GBAR source model for VBR videoconferences," IEEE/ACM Transactions on Networking, vol. 5, no. 4, pp. 554-560, Aug 1997. 